\title{
A DEVELOPMENT OF AUTOMATIC TUNING FOR HIGH ENERGY BEAMLINES AT HIMAC
}

\author{
M. Torikoshi, K. Noda, E. Takada, H. Tsubuku ${ }^{1}$, S. Kai ${ }^{1}$, T. Katsumata ${ }^{1}$ \\ National Institute of Radiological Sciences, 4-9-1 Anagawa, Inage, Chiba 263, Japan \\ ${ }^{1}$ Accelerator Engineering Corporation, 2-13-1 Konakadai, Inage, Chiba 263, Japan
}

\section{Abstract}

A beamline tuning system based on automatic procedure for steering a beam with observing the beam position has been under development for the transport of high energy beams at HIMAC. A daily beamline tuning for radiotherapy and for most biological experiments is carried out to precisely transport the beam to beam delivery devices. This system replays the daily procedure automatically. A beam is steered using steering magnets in accordance with the deviation of beam positions from intended beam positions. How much the beam positions shift due to adjusting a steering magnet is determined by the beam optics design. Therefore, if correlation between deflection angles at the steering magnets and variations in the beam position measured by wire-grids are known, the deflection angles can be calculated by solving linear simultaneous equations. The correlation was measured and compared with those calculated. Most of them are in good agreement. At present, the beamline tuning for delivering beams to the biological experiment room is finished in about 10 minutes automatically.

\section{INTRODUCTION}

A heavy ion medical accelerator, HIMAC, has been providing carbon beams for cancer radiotherapy since June of 1994[1]. The beams extracted from synchrotron rings are transported to three treatment rooms, a biological experiment room and a physics-general experiment hall through high energy beam transport lines (HEBT). The beamline is changed from one to another by only exciting or deexciting switching magnets without tuning the beamlines to change the treatment room in less than 5 minutes. The beamlines are tuned only a few times a day, when the accelerated beam energy or the accelerated ion is changed. The beamline tuning for the radiotherapy and most biological experiments is done in fixed forms, but the beam position, a centroid of a beam profile, should be finally positioned on a central axis of a beam delivery system within in a precision of $\pm 2.5 \mathrm{~mm}$ [2]. At present, two operators are engaged in the operation of the HEBT beamline tuning for the radiotherapy. We want, however, one operator to tune the beamline efficiently. Especially, since the HIMAC has two synchrotron rings, the HEBT beamlines are divided into two systems, a horizontal beamline system (LBT) and a vertical beamline system (UBT) [3]. Each system is operated independently. If we have, therefore, an automatic beam tuning system, only one operator can tune two beamlines of both systems, respectively, at the same time. In this paper, we report our automatic tuning system which is under development for the HEBT system.

\section{PRINCIPLE OF THE AUTOMATIC TUNING}

In everyday's beamline tuning for the radiotherapy and for most of the biology experiments, we basically steer the beam using steering magnets and sometimes bending magnets with monitoring the beam positions using wiregrids. The goal of the beamline tuning is to position the beam on the designed points in the beam delivery devices. The points are defined by two wire-grids, one is located upstream of the isocenter of a treatment room or the biology experiment room, and another is in front of the entrance of the room. The beam profile is not necessary to be adjusted insofar as the profile is approximately round shape in the rooms where horizontal and vertical components of the $\beta$ function were designed to be a same value. Therefore, quadrupole magnets are usually excited in designed strengths.

The principle of the beamline tuning is to steer the beam with the steering magnets and move it to a required position at each wire-grid as shown in Fig. 1. Insofar as a

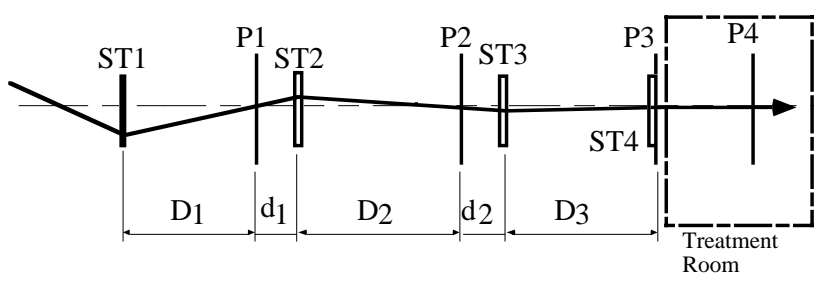

Figure 1: A typical configuration of steering magnets and wire-grids for observation of beam positions. ST's and P's mean steering magnets and wire-grids, respectively.

condition, $d / D<1$, is satisfied, incident angles of the beam with respect to a required path converge, where $\mathrm{D}$ is a distance between a steering magnet and a downstream wire-grid, and $\mathrm{d}$ is a distance between a wire-grid and a next steering magnet. If we know how much the beam position varies at a wire-grid for changing the excitation current of a steering magnet, we can calculate the excitation current of a steering magnet in order to move the beam to a required position at the wire-grid. Once beam optical design has been fixed, the variation in 
a beam position at an arbitrary wire-grid with respect to the variation in a deflection angle at an arbitrary steering magnet upstream of the wire-grid is calculated using transfer matrix equation as the following,

$$
\left(\begin{array}{c}
u \\
u^{\prime}
\end{array}\right)=\left(\begin{array}{ll}
M_{11} & M_{12} \\
M_{21} & M_{22}
\end{array}\right)\left(\begin{array}{l}
u_{0} \\
u_{0}^{\prime}
\end{array}\right)
$$

where $\mathrm{u}_{0}$ and $\mathrm{u}_{0}{ }^{\prime}$ are a beam position and a slope of the beam's trajectory at a steering magnet, respectively. The $\mathrm{u}$ and $\mathrm{u}^{\prime}$ are a position and a slope at a wire-grid, respectively. When the beam is deflected by a steering magnet in different strengths without altering the beam position, we can observe beam positions $\mathrm{u}_{\mathrm{A}}$ and $\mathrm{u}_{\mathrm{B}}$ at $\mathrm{a}$ downstream wire-grid, which are corresponding to the strengths A and B of a steering magnet. They are described as follows,

$$
\begin{aligned}
& \mathrm{u}_{\mathrm{A}}=\mathrm{M}_{11} \mathrm{u}_{0}+\mathrm{M}_{12} \mathrm{u}_{\mathrm{A} 0}{ }^{\prime} \\
& \mathrm{u}_{\mathrm{B}}=\mathrm{M}_{11} \mathrm{u}_{0}+\mathrm{M}_{12} \mathrm{u}_{\mathrm{B} 0}{ }^{\prime}
\end{aligned}
$$

Subtracting the second equation from the first, the ratio of the variation in the beam position to the variation in the deflection angle is derived as follow,

$$
\alpha=\left(\mathrm{u}_{\mathrm{A}}-\mathrm{u}_{\mathrm{B}}\right) /\left(\mathrm{u}_{\mathrm{A} 0}{ }^{\prime}-\mathrm{u}_{\mathrm{B} 0}{ }^{\prime}\right)=\mathrm{M}_{12}
$$

where $\alpha$ is the ratio in the unit of $\mathrm{mm} / \mathrm{mrad}$. Assuming a linear relation between the deflection angle and the excitation current, the $\alpha$ can be converted to the ratio of the position to the excitation current with respect to a certain magnetic rigidity of the beam, which we call it $\eta$. Then, if we know the differences between the initial beam positions and the required positions, we can calculate the current variations of steering magnet by solving the following matrix equation inversely,

$$
\left(\begin{array}{c}
\Delta \mathrm{u}_{1} \\
- \\
- \\
\Delta \mathrm{u}_{\mathrm{n}}
\end{array}\right)=\left(\begin{array}{cccc}
\eta_{11} & 0 & 0 & 0 \\
- & - & - & - \\
- & - & - & - \\
\eta_{\mathrm{n} 1} & - & - & \eta_{\mathrm{nn}}
\end{array}\right)\left(\begin{array}{c}
\Delta \mathrm{I}_{1} \\
- \\
- \\
\Delta \mathrm{I}_{\mathrm{n}}
\end{array}\right)
$$

where $\eta_{i j}$ is the ratio of the position variation of an i-th wire-grid to the current variation of a $\mathrm{j}$-th steering magnet. $\eta_{\mathrm{ij}}=0$ for $\mathrm{i}<\mathrm{j} . \quad A \Delta \mathrm{I}_{\mathrm{j}}$ is the current variation to be solved and a $\Delta \mathrm{u}_{\mathrm{j}}$ is the deviation of the beam position from the required beam position at the $i$-th wire-grid. A case of $i=j$ indicates a relation between a steering magnet and the nearest wire-grid which is immediately downstream from the steering magnet, such as the relation of ST1 and P1 shown in the figure 1. As shown in the equation (1), the $\alpha$ is equal to the transfer matrix element $M_{12}$ which is described as follows:

$$
\mathrm{M}_{12}=\sqrt{\beta_{\mathrm{ST}} \beta_{\mathrm{WG}}} \sin \Psi_{\mathrm{ST}_{-} \mathrm{WG}}
$$

where $\beta_{\mathrm{WG}}$ and $\beta_{\mathrm{ST}}$ are values of a $\beta$ function at the wiregrid and the steering magnet, respectively, and the $\Psi_{\text {ST-WG }}$ is a phase advance from the steering magnet to the wiregrid. So the $\alpha$ 's can be calculated. In our tuning system, however, the measured values are used.

The last steering magnets (ST4 in the figure) is only about $20 \mathrm{~cm}$ away from the wire-grid (P4) which is in front of the room. Therefore, if the beam is in the center of the wire-grid, the beam can be aligned to the required path in the room by only steering the beam with the last steering magnets with a negligibly small change in the beam position at the wire-grid.

\section{PERFORMANCE OF THE AUTOMATIC TUNING SYSTEM}

At present, the automatic tuning process is divided into three parts. Fig. 2 shows the configuration of steering magnets and wire-grids of the vertical beamlines with omitting quadrupole magnets in order to simplify.

The first part is to align a beam with a path of the HEBT beamline which is defined by the first two wiregrids with help of steerers of a synchrotron extraction

\section{Vertical Beamlines}

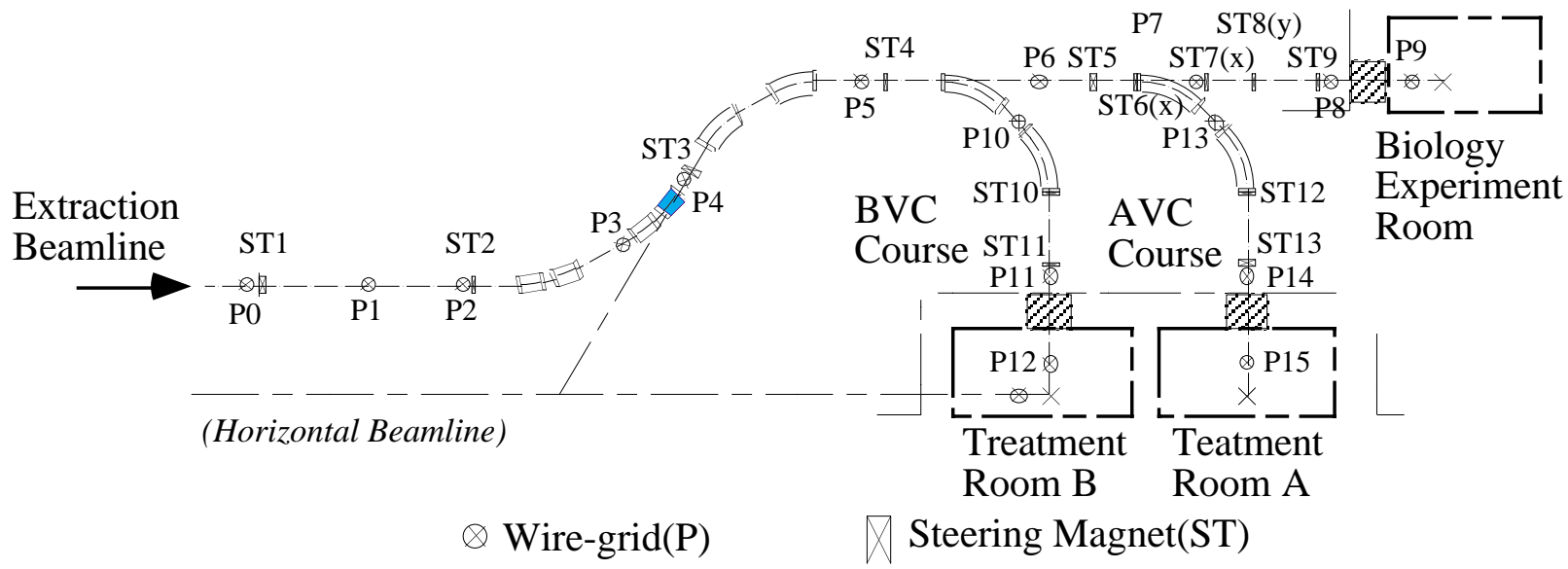

Figure 2: The configuration of steering magnets, wire-grids and bending magnets in vertical beamlines of the high energy beam transport system at the HIMAC. ST and P mean a steering magnet and a wire-grid, respectively. 
beamline. In this part, the beam is adjusted to be positioned on the centers of the two wire-grids, P1 and $\mathrm{P} 2$, using steerers of the extraction beamline as well as the first pair of the steering magnets, ST1. But each beamline is controlled by the different computer one another, and the HEBT's computer cannot directly control the steerers of the extraction beamline. The HEBT's computer adjusts the excitation currents of the ST1, but it only informs us how much change the excitation currents of the steerers of the extraction beamline in order to adjust the beam position in the centers of the wire-grids $\mathrm{P} 1$ and $\mathrm{P} 2$. Therefore, this part is said to be a computer aided tuning rather than the automatic tuning.

The second part is to automatically transport the beam to the entrance of the treatment room or the biology experiment room. In this part, the wire-grids and the steering magnets which are located along the beamline downstream of the P2 are in use. In a case of BIO course, the beam positions at the wire-grids of P3 $\sim$ P8 are measured first. In this process, the $\Delta u$ 's are immediately obtained. Then the $\Delta \mathrm{I}$ 's are derived by inversely solving the equation (2). We can arbitrarily select which wire-grids or steering magnets are in use, and how many times this part is repeated. One of $15^{\circ}$ deflection bending magnets can be also involved to steer the beam vertically as an auxiliary steerer. We can also set values as parameters for the position in which the beam should go and for the tolerance of positioning the beam around the required position at each wire-grid. In most cases, $\pm 0.5 \mathrm{~mm}$ is set as the tolerance. When the repetition number is set to be more than one time, this part is repeated until the beam position coincides with the required position within the assigned tolerance at every wire-grid. If it has not completed within the repetition times, the computer informs the operators it by indicating "TIME OUT". The

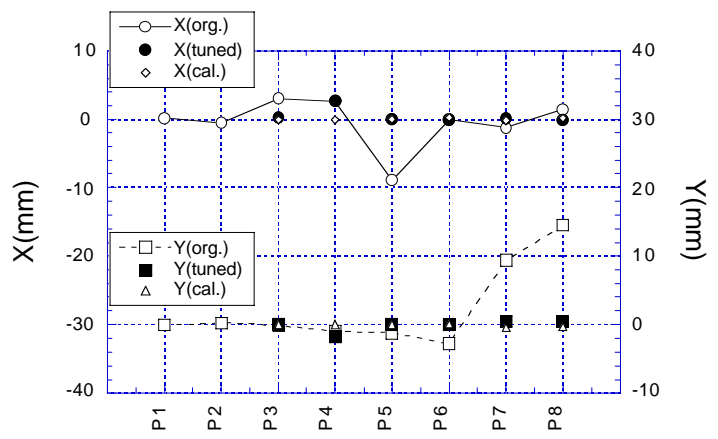

Figure 3: Results of automatic tuning BIO course which transfer beams to the biology experiment room for a carbon beam of $290 \mathrm{MeVA}$. The abscissa is indicating the wire-grids. The open circles and squares show the beam position before the tuning. The black circles and squares show those after the tuning of the second part. Every beam position is on the center of the wire-grid within \pm $0.5 \mathrm{~mm}$ except at the wire-grid $\mathrm{P} 4$ which is out of use in the tuning. wire-grid P4 and the steering magnet ST6 are usually out of use. The former is mainly used for tuning the beamline which joints the vertical beamline and the horizontal beamline. The steering magnet ST6(x) is only manually used, when the horizontal tuning is out of range of the steering magnet ST5. The vertical deflection steering magnets ST8(y), which should be paired with ST7(x) near the wire-grid P7, separates from the ST7(x), because of too tight space near the P7. But the configuration of them still satisfies the condition for the convergence mentioned above. A result of the automatic tuning for the BIO course is shown in Fig. 3, which has been done in 10 minutes.

The final part is to steer the beam using the pair of the most downstream steering magnet and to position the beam on the center of the wire-grid located in the treatment room or the biology experiment room. Taking the BIO course as an example again, the beam has been adjusted to be in the center of the wire-grid P8 in the second part. Since the pair of the most downstream steering magnets ST9 are very close to the wire-grid P9, both of the locations can be regarded as being same. Only moving the beam position to the center of the wire-grid P9 by using the steering magnets ST9, the beam should be aligned on the required path of the irradiation devices for the biology experiments or the treatment, which is defined by the P8 and P9.

\section{SUMMARY}

These scenarios of the automatic tuning the other beamlines for the treatments, such as AVC course, are same. The first and second parts are well used, for especially tuning the BIO course which has the longest beamline. This system transports the beams to the treatment rooms and biology experiment room with sufficiently good precision for the radiotherapy and the biology experiments, while the operator does not need a special knowledge of the beam transportation. The beam transportation for the most treatments is done in fixed forms, so the automatic tuning system is easily applicable and very convenient.

\section{REFERENCES}

[1] S. Yamada: Commissioning and performance of the HIMAC medical accelerator", Proc. of the 1995 IEEE PAC, Vol.1 pp.5.

[2] T. Kanai, et al.: 'HIMAC beam delivery system physical characteristics-', Proc. of XXI PTCOG Meeting, Chiba, 1994, HIMAC Rep. 008, 1994, pp. 26.

[3] M. Torikoshi, et al.: 'Control system of a high energy beam transport system of HIMAC', Proc. of the 10th Symp. on Accelerator Science and Technology, Hitachi Naka, 1995, pp. 306. 